7th International Workshop on Astronomy and

Relativistic Astrophysics (IWARA 2016)

International Journal of Modern Physics: Conference Series

Vol. 45 (2017) 1760027 (7 pages)

(C) The Author(s)

DOI: $10.1142 / S 2010194517600278$

\title{
Simulations of the Neutron Star Crust
}

\author{
Stefan Schramm and Rana Nandi \\ Frankfurt Institute for Advanced Studies (FIAS) \\ Ruth-Moufang-Str. 1, D-60438 Frankfurt am Main, Germany \\ schramm@fias.uni-frankfurt.de,nandi@fias.uni-frankfurt.de
}

Published 15 August 2017

\begin{abstract}
The properties of the neutron star crust are crucially important for many physical processes occurring in the star. For instance, the crustal transport coefficients define the temperature evolution of accreting stars after bursts, which can be compared to observation. Furthermore, the structure of the inner crust can modify the neutrino transport through the matter considerably, significantly impacting the dynamics of supernova explosions. Therefore, we perform numerical studies of the inner crust, and among other aspects, investigate the dependence of the pasta phase on the isospin properties of the nuclear interactions. To this end we developed an efficient computer code to simulate the inner and outer crust using molecular dynamics techniques. First results of the simulations and insights into the crust-core transition are presented.
\end{abstract}

Keywords: Neutron Star, Star Crust, Molecular Dynamics.

PACS numbers: 97.60.Jd; 26.60.-c; 52.65.Yy

\section{Introduction}

Many properties of strong interaction theory are still unknown. In particular, there are large experimental efforts to investigate strongly interacting matter at high temperature and varying density in ultra-relativistic heavy-ion collisions. Complementary to this effort the study of neutron stars allows for deducing or at least constraining the behaviour of very dense and mostly cold matter. Interpreting neutron star observations frequently requires knowledge not only of the high-density core but also of the about $1 \mathrm{~km}$ thick crust that surrounds it. Here, the outer crust consists of a sequence of more and more neutron rich nuclei forming a lattice embedded in a gas of electrons. At higher densities beyond the neutron drip, nuclei are also immersed in a gas of free neutrons in the inner crust. The properties of this crust strongly influence various aspects of neutron star physics like

This is an Open Access article published by World Scientific Publishing Company. It is distributed under the terms of the Creative Commons Attribution 4.0 (CC-BY) License. Further distribution of this work is permitted, provided the original work is properly cited. 


\section{S. Schramm \& R. Nandi}

their cooling behaviour after X-ray bursts, triggered by accretion from a companion star. ${ }^{1}$ The crust samples strong interaction, or nuclear, physics at high isospin because of the increasing fraction of neutrons towards the core region. Aside from the many unknown properties of matter in the core, also the behaviour of strong interactions at very large isospin asymmetries is by far not fully understood. This lack of knowledge already exists at saturated and lower nuclear matter densities. An indication for this is the extreme uncertainty of the position of the neutron drip line, particularly when considering heavier nuclei. This is illustrated by a calculation of the binding energy of uranium isotopes using a set of common interactions based on Skyrme, relativistic mean field and chiral effective models. Figure 1 shows the strongly diverging results. The minimum of the binding energy with respect to neutron number $\mathrm{N}$ indicates the position of the neutron drip. While some parameter sets show particle-stable isotopes only up to $\mathrm{N}=182$ (e. g. $\chi_{M}$, not shown in the plot $^{2}$ ), NL-Z2 (see Ref. ${ }^{3}$ ) (and other parameter sets) support nuclei with up to 258 neutrons, leading to an uncertainty of the drip point by more than 70 neutrons ${ }^{4}$ !

\section{QMD Simulations of the Neutron Star Crust}

In order to understand the dynamics of the inner crust with its many nearly degenerate minima we adopt a semiclassical molecular dynamics approach of simulating the crustal properties. In such an approach the nucleon wave functions are represented by Gaussian wave packets with time-independent widths. The time evolution of the centers of the wave functions is determined by a many-body Hamiltonian. In first studies we adopt the effective interaction introduced in Ref., ${ }^{5}$ augmented by non-linear isospin dependent forces, in order to study possible isospin effects on the

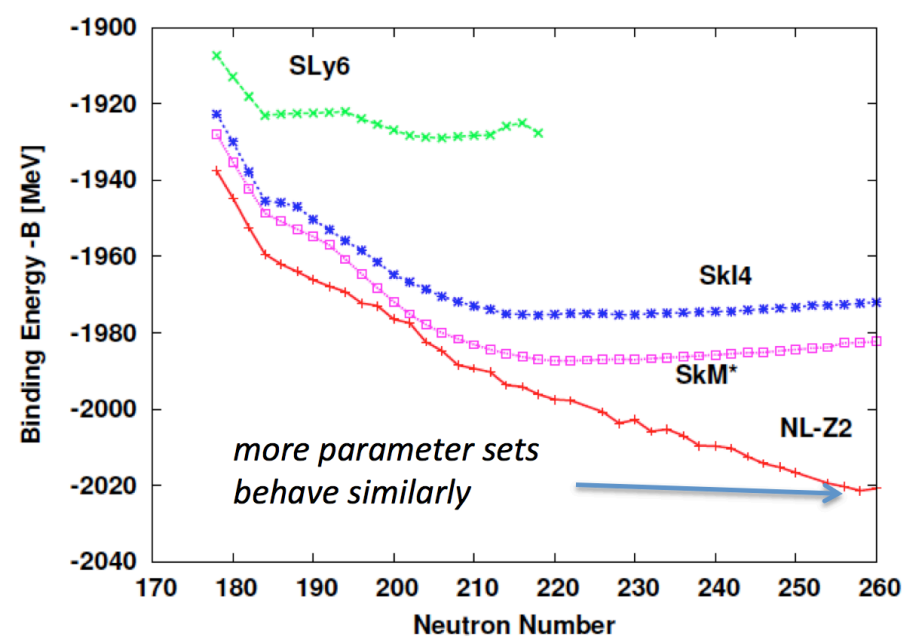

Fig. 1. Binding energy of uranium isotopes for various models as function of neutron number. 


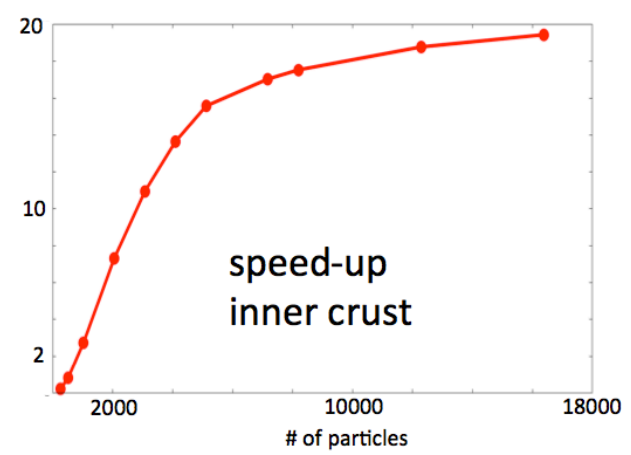

Fig. 2. Speed up of GPU-based calculation compared to a 24-core CPU execution.

results. The general structure of the Hamiltonian reads

$$
H=T+V_{\text {Pauli }}+V_{\text {Skyrme }}+V_{\text {sym }}+V_{\text {MD }}+V_{\text {Coul }},
$$

including the kinetic energy $T$, an effective Pauli potential $V_{\text {Pauli }}$, mimicking Pauli blocking, momemtum-independent and -dependent Skyrme-type interactions $V_{\text {Skyrme }}, V_{\mathrm{MD}}$, isospin-dependent interactions $V_{\text {sym }}$, as well as the Coulomb interaction $V_{\text {Coul }}$. For an explicit description of the terms, see Ref. ${ }^{6}$

In order to sample systems with many particles over large simulation times an efficient implementation of the dynamics governed by Eq. (1) is required. To that end we ported our simulation code to a graphics processor (GPU) environment, leading to substantial speed-ups as can be seen in Fig. 2.

In order to test the basic validity of our code and the implemented forces, we calculate the binding energy of a range of nuclei from ${ }^{40} \mathrm{Ca}$ to ${ }^{208} \mathrm{~Pb}$. In Fig. 3 one can see that the energies agree quite well with experimentally measured results, given the limits of a molecular dynamics - type approach.

Continuing from this result we study the isospin dependence of nuclear matter by changing the relative numbers of protons and neutrons in the simulation box. One straightforward, but quite important result of such a simulation is shown in Fig. 4. Here, the energy per particle is plotted as function of the asymmetry $\delta=\frac{\rho_{p}-\rho_{n}}{\rho_{p}+\rho_{n}}$ at saturation density.

The various curves correspond to different choices of the isospin force parameters. ${ }^{6}$ The left panel shows results to the simulation data with a quadratic function, whereas the right-hand panel contains the same data using a fit including a fourthorder dependence on $\delta$. One can observe significant deviations from the often-used simple quadratic asymmetry dependence of the energy, demonstrating the need for higher-order terms in order to describe the asymmetry energy over a larger range correctly. 


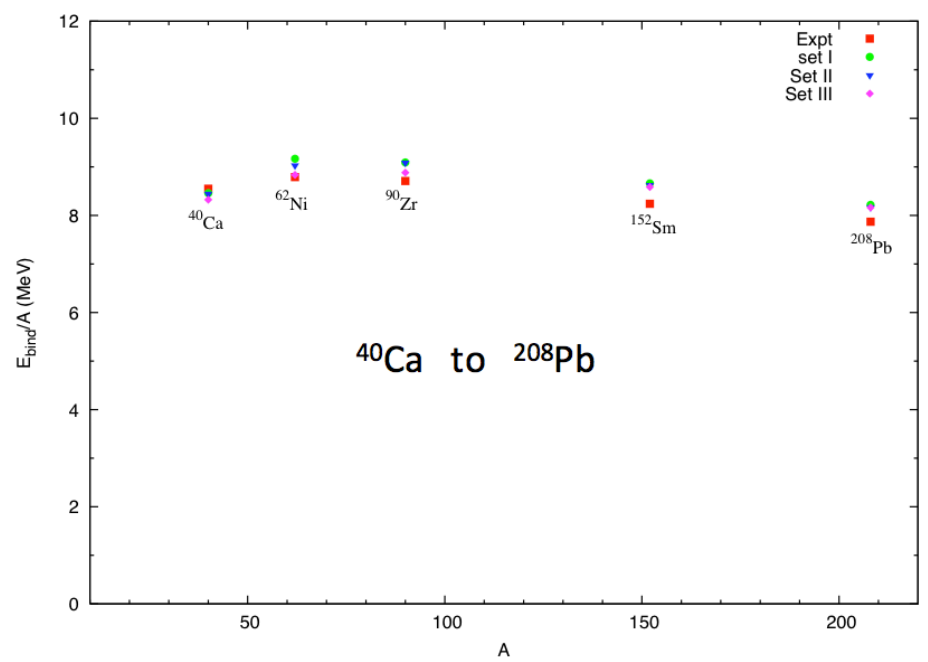

Fig. 3. Binding energies per nucleon of a set of nuclei as calculated in the QMD calculation for different parameter sets. For comparison experimental values are also indicated.
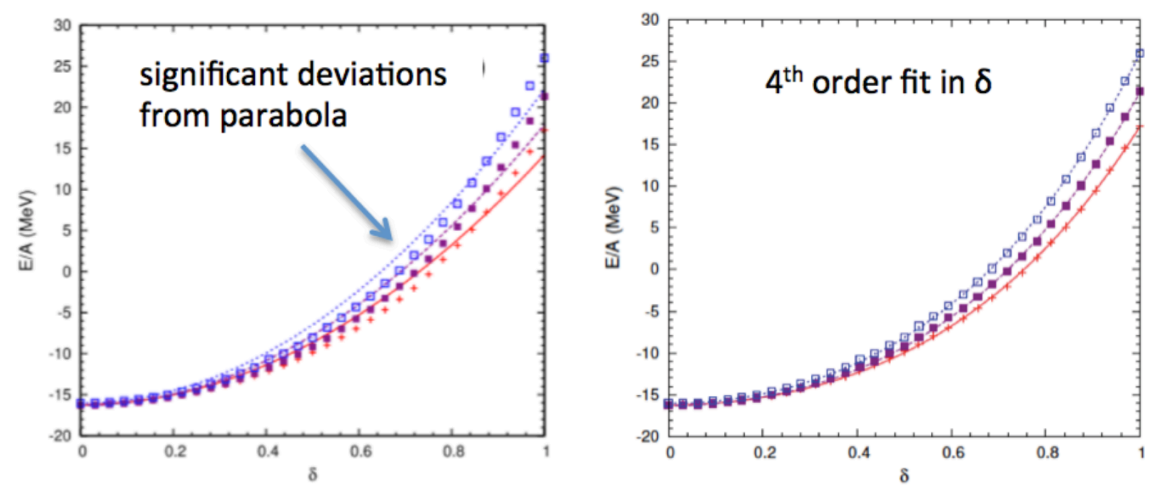

Fig. 4. Asymmetry energy as function of density compared to quadratic fit (left figure) and to a fit up to fourth order in the asymmetry parameter $\delta$ (right figure).

A very interesting regime for crust simulations is the range where the structured inner crust melts into a largely homogeneous core matter of neutrons, protons, and electrons. Here, the interplay of surface and Coulomb energy can generate unusual nuclear shapes, the so-called pasta phases, leading to cylindrical and plane-shaped structures and, at higher densities, to analogous inverted shapes of voids in the dense matter. ${ }^{7,8}$ The numerical simulations in fact show such structures as is illustrated in Fig. 5.

Zooming into densities close to the onset of the homogenous core, this transition can be observed by computing two-particle correlations as they change with density. 

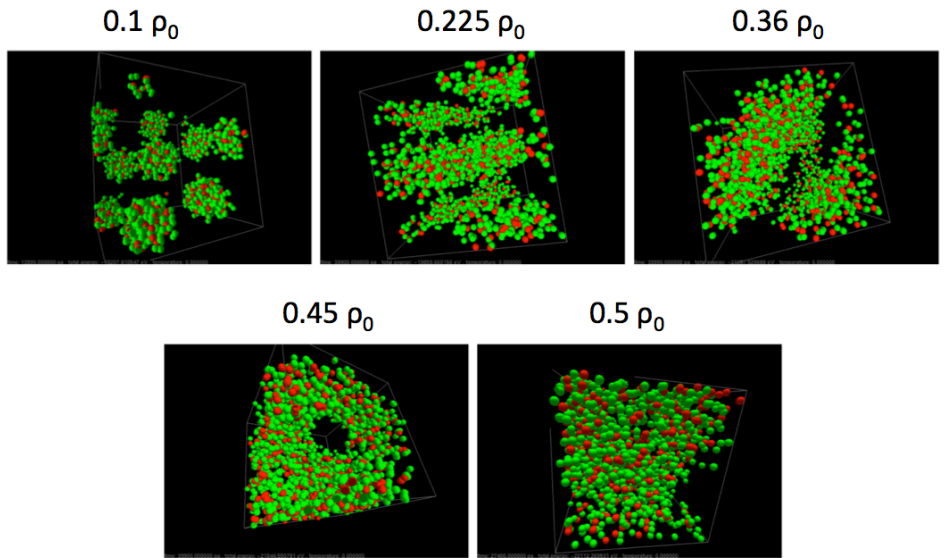

Fig. 5. Snapshots of structures obtained in the QMD simulation for various densities for a protonto-neutron ratio of 0.3 . The green and red symbols correspond to neutrons and protons, respectively.

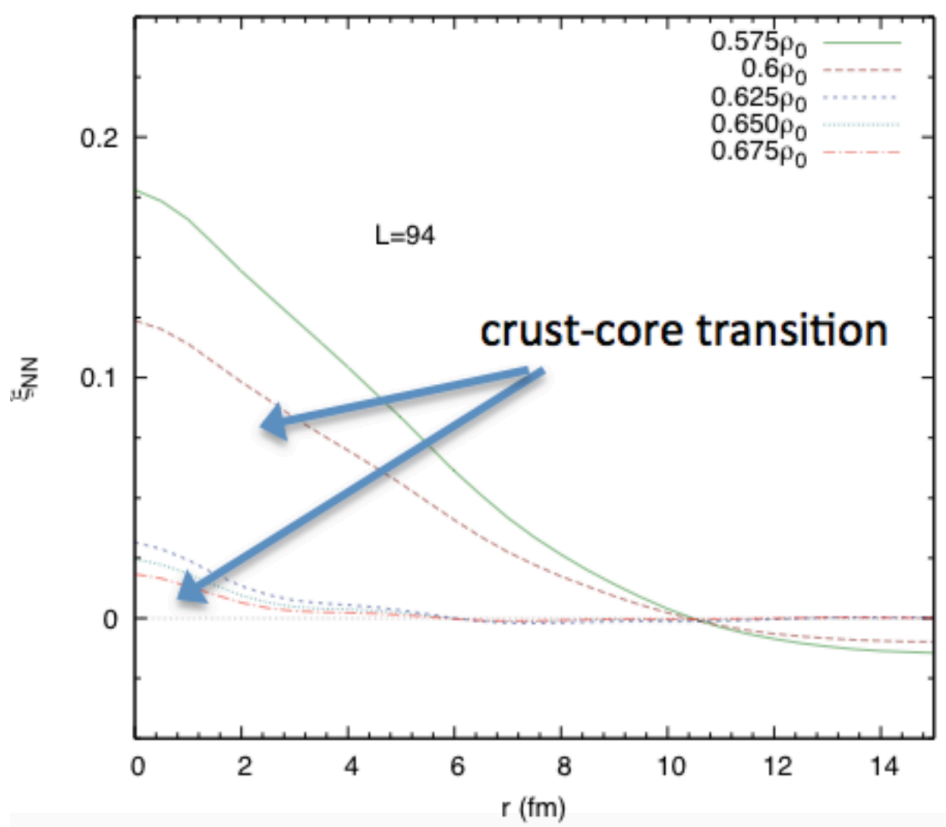

Fig. 6. Two-particle correlation as function of interparticle distance for a set of baryon densities, indicating a rapid transition to a homogeneous core. 

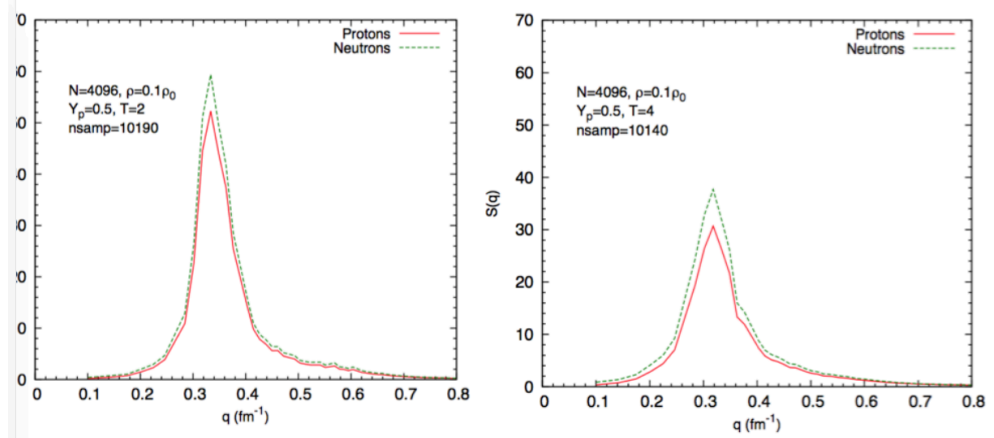

Fig. 7. Proton and neutron structure factor $S(q)$ averaged over the direction of $\mathbf{q}$ as function of momentum for two different temperatures $\mathrm{T}=2 \mathrm{MeV}$ (left panel) and $4 \mathrm{MeV}$ (right panel), respectively. The calculation was performed for symmetric matter including electrons at a baryon density $\rho=0.1 \rho_{0}$.

Fig. 6 shows the correlation $\xi_{N N}(r)$, defined as

$$
\xi_{N N}(r)=\left\langle\triangle_{N}(\mathbf{x}) \triangle_{N}(\mathbf{x}+\mathbf{r})\right\rangle
$$

Here the fluctuation $\triangle_{N}(\mathbf{x})$ of the nucleon density field $\rho_{N}(\mathbf{x})$ is given by

$$
\triangle_{N}(\mathbf{x})=\frac{\rho_{N}(\mathbf{x})-\rho_{\mathrm{av}}}{\rho_{\mathrm{av}}}
$$

with the mean density $\rho_{\mathrm{av}}$ and averaging over the position $\mathbf{x}$ and the direction of r. The results clearly show the rapid suppression of the correlations with density, hinting to a first-order crust-core phase transition at about $\rho=0.65 \rho_{0}$. Changing the isospin parameters of the interactions as done in the calculations for Fig. 4 does not significantly change this behaviour as discussed in Ref., ${ }^{6}$ which is in contrast to other reported studies. ${ }^{9}$

As a final preliminary result of the numerical simulations, Fig. 7 presents the structure factor $S_{i}()$ for protons and neutrons $(\mathrm{i}=\mathrm{p} ; \mathrm{n})$, defined as

$$
S_{i}(\mathbf{q})=<\rho_{i}^{*}(\mathbf{q}) \rho_{i}(\mathbf{q})>
$$

with the Fourier-transformed densities $\rho_{i}(\mathbf{q})$. Here, the shape of the momentum dependence of the form factor reflects the clusters and their sizes in the system. This function directly enters the expressions for various transport properties of the stellar matter, like conductivities and the shear viscosity, which have direct relevance for the cooling behaviour of accreting neutron stars and neutrino emission including potential reheating of supernova matter. ${ }^{10}$ The numerical calculation of these transport properties is currently in progress and will be reported on in the near future. 


\section{Acknowledgments}

The authors acknowledge support by the LOEWE program HIC for FAIR. The numerical calculations were performed on the LOEWE-CSC cluster at the Goethe University in Frankfurt.

\section{References}

1. R. L. Merritt, E. M. Cackett, E. F. Brown, D. Page, A. Cumming, N. Degenaar, A. Deibel, J. Homan, J. M. Miller and R. Wijnands, Ap. J. 833, 186 (2016).

2. S. Schramm, Physical Review C 66, 064310 (dec 2002).

3. M. Bender, K. Rutz, P.-G. Reinhard, J. Maruhn, and W. Greiner, Phys. Rev. C 60, 034304 (1999).

4. S. Schramm, D. Gridnev, D. V. Tarasov, V. N. Tarasov, and W. Greiner, International Journal of Modern Physics E 21, 1250047 (apr 2012).

5. T. Maruyama, K. Niita, K. Oyamatsu, T. Maruyama, S. Chiba, and A. Iwamoto, Physical Review C 57, 655 (feb 1998).

6. R. Nandi and S. Schramm, Physical Review C 94, 025806 (aug 2016).

7. D. Ravenhall, C. Pethick, and J. Wilson, Physical Review Letters 50, 2066 (jun 1983).

8. M.-a. Hashimoto, H. Seki, and M. Yamada, Progress of Theoretical Physics 71, 320 (feb 1984).

9. S. S. Bao and H. Shen, Physical Review C 89, 045807 (apr 2014).

10. C. J. Horowitz and D. K. Berry, Physical Review C 78, 035806 (sep 2008). 